\title{
Sensation, culture, sensibilité
}

Jean-Bernard Paturet

\section{OpenEdition}

\section{Journals}

Édition électronique

URL : http://journals.openedition.org/trema/1915

DOI : 10.4000/trema.1915

ISSN : 2107-0997

\section{Éditeur}

Faculté d'Éducation de l'université de Montpellier

\section{Édition imprimée}

Date de publication : 1 avril 1997

Pagination : 5-18

ISSN : 1167-315X

\section{Référence électronique}

Jean-Bernard Paturet, « Sensation, culture, sensibilité », Tréma [En ligne], 11 | 1997, mis en ligne le 01 avril 1997, consulté le 30 avril 2019. URL : http://journals.openedition.org/trema/1915 ; DOI : 10.4000/ trema.1915

Ce document a été généré automatiquement le 30 avril 2019.

Trema 


\title{
Sensation, culture, sensibilité
}

\author{
Jean-Bernard Paturet
}

\section{Introduction et problématique}

1 Loin d'être l'exposé cohérent d'un système de pensée, la réflexion sur les problèmes d'éducation à la sensibilité est plutôt une démarche sur la question, une exposition en perpétuel déséquilibre. Elle soulève souvent plus d'interrogations qu'elle n'en résout. $\mathrm{A}$ l'instar des péripatéticiens, la méditation sur la sensibilité est une invitation à la " promenade ». Devenir un « ego promenans » et pas seulement un « ego cogitans » voilà ce que suggère Michel Malherbe dans ses Trois essais sur le sensible.

"L'ego promenans ", dit-il, " cherche d'abord son pas : il le règle sur son souffle et puisque le terrain est varié il raccourcit ou retarde son allure pour préserver le bonheur tranquille et ajusté de sa marche. Il évite les rythmes fatigants et monotones, telle la dialectique, où il faut à chaque fois faire un pas en arrière. Il évite tout autant les chemins déductivement tracés au préalable, avec arrêt systématique et pause récapitulative. Quand il rencontre une belle idée, il l'observe sans la cueillir, pour la laisser fraîche au regard d'un autre promeneur. Il n'aime pas les raisonnements raides, les voies directissimes, les triomphes nécessitant [...] son grand plaisir est certainement de prendre des chemins de traverse, an gré de sa fantaisie philosophique, et le soir venu, quand la fatigue incline à la réflexion, de découvrir que les hasards de la marche lui ont assuré un parcours en définitive cohérent, que décidément, il y a de la raison dans toutes ces pérégrinations, mais une raison aimable et paisible ».

Leçon de philosophie, leçon d'humilité aussi mais surtout invitation à entrer dans une démarche singulière et dans une méthodologie de l'observation originale qui se laisse porter et aspirer par les découvertes. Devenir « ego promenans » c'est suivre les chemins de traverse au gré d'une observation souple, c'est être à l'écoute de l'environnement, c'est être disposé à la rencontre et être disposé à s'exposer soi-même en laissant place au libre jeu des sens pour que se dévoile la sensibilité et que se découvre la multiplicité du sens...

Lorsque l'on évoque la question de «la sensibilité ", l'on s'aperçoit très vite que la langue (la langue française, en tout cas) cache sous ce terme une multitude de signifiés : «la 
douleur est-elle encore sensible? », «je suis sensible à un beau coucher de soleil », « cet appareil est ultra sensible» ou encore "cet enfant est trop sensible». D'une façon générale, la sensibilité serait ce qui renvoie au plaisir ou à la douleur avec des degrés dans la finesse de l'appréhension de cette souffrance ou de ce plaisir. Elle se présenterait donc, a priori, comme une pure subjectivité. Ce que j'éprouve (mon vécu, comme l'on dit) ne l'est bien entendu que pour moi seul, dans le moment de l'ici et maintenant où je le vis même si d'autres peuvent partager par ailleurs une expérience identique. La sensibilité, en terme philosophique, serait donc ce qui s'oppose à l'intelligibilité qui, elle, au contraire, relève de l'universel par le truchement de la rationalité et du discours. Depuis Platon, la philosophie a toujours opposé sensible et intelligible et l'on connaît bien « la symbolique de la ligne» du livre de La République qui les caricature d'une manière radicale, laissant le sensible dans le monde de l'illusion, tandis que l'intelligible est celui de la vérité.

Cependant, si la sensibilité est, a priori, subjectivité et singularité, ne sommes-nous pas en droit de nous demander si nous ne tombons pas dans une « aporie » fondamentale lorsque nous cherchons à " éduquer » la sensibilité ? Car n'y a-t-il pas toujours, sous forme plus ou moins déguisée, une prétention à l'universel, dans tout acte éducatif? N'y a-t-il pas toujours une tentative cachée de normalisation? On essaiera donc de mettre cette réflexion en travail et de repérer quelles sont la place et l'importance de l'apprentissage de la sensibilité dans notre culture.

\section{Sensibilité et perception organique}

5 Pour avancer dans notre méditation (Heidegger oppose la raison calculante et dominante à la raison méditante) commençons par nous référer à plusieurs expériences. Il m'a été donné au cours d'une recherche, il y a quelques années, de rencontrer des aveugles-nés et des personnes devenues aveugles. Réfléchissant sur ce que les uns et les autres sentaient, nous nous sommes arrêtés sur deux petites phrases pour essayer de comprendre ce qu'il en était de la sensation : «le ciel est bleu » - « le soleil est chaud ». Lorsque nous utilisons l'expression : «le ciel est bleu », nous avons une image visuelle, cette image est celle d'un voyant, car pour lui le ciel se voit et le bleu aussi. Mais l'aveugle-né n'en a absolument aucune représentation, il ne "voit » ni le ciel ni la couleur. Dans un très beau livre, Les couleurs de la nuit, Gilbert Siboum, aveugle de naissance, se demande: "Comment imaginer un univers qui n'est fait que de mots, qui ne peut m'être révélé que par eux, alors que pour moi, il ne peut s'exprimer en image?", et quelques pages plus loin, il ajoute : « la couleur fait partie de la vision et je continuais à me demander : voir, qu'estce-que c'est? Quand Gérard » [son ami] "me décrit quelque chose, il ne sait pas ce que cela évoque pour moi, et j'ignore ce que cela représente pour lui » (p. 177). Le mot exprimant une image visuelle n'est pas, par conséquent, "compréhensible» pour l'aveugle de naissance, sinon par une connaissance intellectuelle ou abstraite. Car comme les aveugles-nés le disaient justement : « nous savons que le ciel est bleu parce qu'on nous l'a appris, mais nous ne savons pas ce que cela représente.» Quant à la seconde expression, «le soleil est chaud», il ne s'agit plus pour l'aveugle-né d'une impossible association d'un mot à une représentation insignifiante pour lui, mais d'une expérience vécue, d'une sensation éprouvée par lui lorsqu'il s'expose au soleil. Même si les mots continuent à exprimer une image visuelle, l'expérience de la chaleur solaire est bel et bien éprouvée. 
6 La seconde expérience à laquelle se référer est celle des daltoniens dont on sait qu'ils ne perçoivent pas certaines couleurs et que par conséquent ils confondent en particulier le vert et le rouge. L'analyse montre que l'œil est un organe récepteur composé de photorécepteurs: les bâtonnets et les cônes. Les bâtonnets interviennent peu dans le repérage des couleurs, par contre, les cônes sont sensibles aux couleurs : cônes verts pour le vert, cônes rouges pour le rouge, cônes bleus pour le bleu. L'absence d'une catégorie de cônes produit donc une anomalie.

7 Une première conclusion s'impose alors. La sensibilité est liée au système de perception organique. La carence d'un organe entraîne une impossibilité de sentir tel ou tel aspect du monde environnant. Toute réflexion sur la sensibilité se doit donc de travailler sur la notion même de sensation et sur la façon dont le système physiologique fonctionne. Nous essaierons par conséquent de le faire dans une première partie.

\section{Sensibilité et culture sociale}

Mais revenons pour l'instant à notre problématique et intéressons-nous à une autre expérience rapportée par les auteurs du livre Voir et nommer les couleurs (Nanterre, 1978). Lorsque Newton s'intéresse à la lumière, il réussit à décomposer la lumière blanche au moyen d'un prisme. Ceci lui permet alors de démontrer la nature corpusculaire de la lumière et le caractère propre et indécomposable des couleurs fondamentales. Or, on peut remarquer fort justement que dans sa démonstration originale devant la Société royale des Sciences de Londres (1671), Newton n'avait « retenu que cinq couleurs » primaires : rouge, jaune, vert, bleu et violet (i.e. -purple), alors que, dans la publication finale (1728) on trouve sept couleurs primaires (les mêmes, plus orange et indigo). On ne peut, bien sûr, considérer qu'il s'agit d'une erreur. L'hypothèse à retenir serait, plutôt, que Newton voulait compléter sa liste initiale pour que sa théorie de l'analogie entre le son et la lumière soit plus satisfaisante : sept couleurs dans l'arc-en-ciel, sept tons dans l'octave. On voit ici comment la culture précède et oriente la lecture de la sensation. Or si l'on observe les arcs-en-ciel avec quelque attention, il est difficile de voir vraiment les sept couleurs de Newton. D'ailleurs, on sait que la sensibilité de certains peuples de l'Antiquité leur « permettait » de voir seulement trois couleurs, tandis que d'autres perçoivent dans l'arcen-ciel plus d'une dizaine de couleurs.

Une seconde conclusion s'impose alors. La sensibilité est liée au système de représentation sociale (philosophique et scientifique) d'une culture historiquement donnée. Ceci fait dire à Anne Sauvageot que «l'on ne s'interroge jamais suffisamment sur la façon dont une culture, une société le (le monde) visualisent et le rendent visible ». Car, ajoute-t-elle, "s'il n'y avait pas le langage, le monde serait opaque " (Voir et savoirsA. Sauvageot - PUF 1974). Dans ses Etudes de sociologie de l'Art (Denoël, 1970, p. 60) P. Francastel écrit lui aussi quelque chose d'assez proche : « On ne voit que ce qu'on connaît ou du moins ce qu'on peut intégrer dans un système cohérent et par suite déployé dans le temps des représentations significatives». En ce sens, l'œil «innocent» (in :privatif nocere: nuire - innocent signifie ici : qui n'est pas souillé) de l'aveugle ne voit rien, de même que l'œil « innocent » du voyant « inculte » ne voit rien non plus. Whorf soutenait que toute langue contient une sorte de «métaphysique cachée " qui constitue un programme de l'activité mentale et façonne les idées des individus ainsi qu'il l'a montré dans les diverses façons de comprendre et d'appréhender les concepts de temps, d'espace, de haut et de bas entre les sociétés indo-européennes et celle des Indiens Hopi. Chaque 
langue est liée indissolublement à une représentation du monde qui ne peut se concevoir en dehors du champ de cette langue.

\section{Remarques complémentaires} l'usage de ceux qui voient, que les anomalies et l'éducation peuvent pousser à développer davantage tel ou tel de nos sens. Dans notre culture, le sens de la vue est privilégié, mais les aveugles développent une sensibilité de la peau qui leur permet de sentir et qui, disent certains, est une sorte de "vision", tandis que les voyants atrophient l'odorat par exemple en surinvestissant la vue. Andy Warhol regrettait de ne pouvoir conserver les " odeurs », dans ce monde qui privilégie la vision et l'image :

"J'adorais le hall du Paramount Theater à Broadway. Chaque fois que je m'y trouvais, je fermais les yeux, et je respirais à fond» [la mise en caractères gras est de moimême]. "Et puis on l'a démoli. Je peux regarder la photo de ce hall tant que je veux, et alors? Je ne pourrai plus jamais le sentir. J'ai parfois la vision d'un livre de botanique $d u$ futur qui dirait : "le lilas a maintenant disparu. On pense que son parfum était semblable à celui de..." et que pourrait-on dire de plus?"

(Ma philosophie de A à B), Andy Warhol

$C^{\prime}$ 'est pourquoi, dans une deuxième partie, nous nous interrogerons sur le rapport entre langage et sensibilité. Puis dans une dernière partie, nous nous intéresserons au rapport éducation-sensibilité pour tenter de montrer en quoi l'éducation a pour fonction essentielle en ce domaine de donner « les mots pour la dire».

\section{La sensation et la sensible}

De manière générale, la représentation du «mécanisme » de la sensation peut se concevoir sous la forme du schéma suivant :

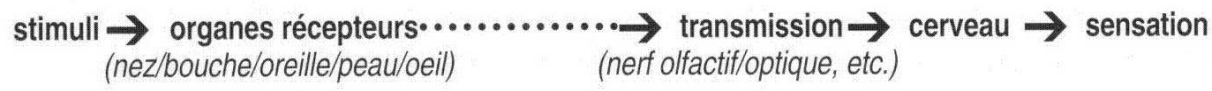

L'œil reçoit des stimuli par la rétine sous la forme de photons absorbés par les photorécepteurs des cellules: les bâtonnets et les cônes (100 photons par seconde saturent les bâtonnets - 10-10 photons par seconde saturent les cônes et provoquent l'éblouissement). Les cônes qui jouent un rôle déterminant dans la couleur sont au nombre de trois catégories: les «bleu », les « vert » et les " rouge ». A partir de ces trois couleurs primaires, toutes les autres peuvent être perçues. Les stimuli sont transmis sous forme d'impulsions électriques au cerveau. L'œil peut alors percevoir les reliefs et les couleurs. Le nez comprend trois centimètres carrés de cellules olfactives. Les stimuli, sous forme de molécules en phase gazeuse de faible poids, parviennent à la muqueuse olfactive (récepteurs) par la voie nasale. Le nez perçoit alors les odeurs et les arômes. La transmission au cerveau se fait ensuite à partir de la muqueuse olfactive par le neuroépithélium olfactif vers le bulbe olfactif (partie spécialisée du cerveau). La bouche reçoit des molécules en solution dans la salive. Les récepteurs sont des cellules épidermiques spécialisées situées sur la langue, les joues, les gencives et le pharynx. En Occident, on retient quatre saveurs : le sucré (zone spécialisée sur la pointe de la langue), le salé (sur les côtés antérieurs de la langue), l'acide (sur les côtés postérieurs de la langue) et l'amer (sur l'arrière de la langue et le pharynx). Notons dès maintenant que d'autres cultures ont 
cinq ou six saveurs. La peau permet de recevoir les stimuli thermiques: le chaud et le froid ou les sensations pseudo-thermiques, les piquants, les irritants (acide), les astringents. Elle permet aussi de repérer la fluidité, l'onctuosité, la dureté.

14 La sensation pourrait donc apparaître comme le donné élémentaire partagé par les "vivants" (en fonction bien sûr du développement de leurs organes sensoriels). La sensation pourrait se définir comme un vécu immédiat des organes sensoriels à leur milieu. Cette réaction sensorielle dépendrait essentiellement de l'organe des sens excités plus que de la nature de l'excitant. Preuve en est qu'un excitant mécanique (par exemple un coup de poing sur l'œil) provoque, ici, une sensation visuelle (voir 36 chandelles) de même qu'un excitant lumineux (lampe électrique) donne à l'œil une sensation visuelle. A l'inverse, si une excitation identique est appliquée à différents nerfs sensitifs, on obtient des sensations différentes. L'excitation mécanique du nerf optique (par exemple la présence d'une tumeur au cerveau) provoque des hallucinations, tandis que l'excitation du nerf acoustique entraîne l'apparition de bruits (hallucinations acoustiques). Ceci tendrait donc à souligner à la fois le caractère physiologique et subjectif de la sensation. La sensation serait alors le mode de réaction des organes sensoriels à des stimuli. Elle n'est pas, par conséquent, le reflet d'un objet du monde extérieur. Elle n'est pas une image de l'excitant pour plusieurs raisons.

Bergson observait déjà qu'« une seconde de lumière rouge qui a la plus grande longueur d'ondes et dont les vibrations sont, par conséquent, les moins fréquentes accomplit quatre cents trillions de vibrations ». Il faudrait plusieurs années pour percevoir chacune de ces vibrations. La sensation apparaît donc comme une synthèse mentale. Elle n'est aucunement une copie de l'excitant, mais une activité de synthèse. Cela signifie que nos organes sensoriels opèrent un choix mais un choix très limité dans toute la gamme des vibrations offertes par la nature. Nous ne traduisons, par conséquent, qu'un nombre très restreint d'aspects du monde. L'abeille, par exemple, est aveugle à la couleur rouge, mais elle perçoit l'ultra violet. L'abeille « voit » quatre catégories de couleurs de l'ultra-violet au vert-jaune, en passant par le bleu violet et le bleu cyan (kuanos = bleu sombre). Le pigeon, quant à lui, se contente de trois catégories de couleurs : bleu-vert, rouge-orange, jaune.

16 Les humains « voient » quatre couleurs fondamentales irréductibles les unes aux autres: bleu, vert, jaune, rouge et il semble établi que les bébés distinguent ces couleurs bien avant l'acquisition du langage. Ces quatre couleurs couramment dénommées " primaires psychologiques » sont fondamentales au niveau des comportements. (cf. le livre déjà cité : Voir et nommer les couleurs). La sensation découpe donc dans l'univers de chaque espèce vivante ce que von Uexküll appelle son « Umwelt », son monde environnant. L'« Umwelt » des humains n'est pas au niveau de la sensation de celui de l'abeille ou du pigeon. La sensation n'est pas, par conséquent, un décalque de l'univers, elle en est déjà un mode de construction que l'être vivant élabore dans son effort d'adaptation. Prenons-en pour preuve les travaux de Jacques Bouveresse dans son livre Langage perception et réalité (Tome I La perception et le jugement). Dans le chapitre «Ce que l'œil dit au cerveau » il reprend un article «ce que l'œil de la grenouille dit au cerveau de la grenouille». Il s'agit dans ce texte de mettre à l'épreuve l'idée selon laquelle,

" on a toujours fait la supposition que l'œil sent principalement de la lumière dont la distribution locale est transmise au cerveau en une espèce de copie par une mosaïque d'impulsions. Supposons que nous soutenions au contraire que l'appareil nerveux dans l'œil est lui-même consacré à la détection de certaines configurations (patterns) de lumière et de leurs changements, correspondant à des relations particulières dans le monde sensible. Si 
cela devait être le cas, les lois que l'on découvre en utilisant de petites taches de lumière sur

la rétine peuvent être vraies et néanmoins, en un certain sens, nous induire en erreur ". immobiles du monde qui l'environne, ou, en tout état de cause, elle ne semble pas concernée. Par conséquent, la grenouille pourrait mourir au milieu d'une provision de nourriture accessible, si cette nourriture n'est affectée d'aucun mouvement. Pour s'alimenter la grenouille tente toujours de capturer n'importe quel objet qui passe à sa portée, de la taille d'un ver ou d'un insecte, pourvu qu'il bouge de la même façon. Elle peut alors être mystifiée facilement par n'importe quel objet (hameçon par exemple) en mouvement de dimensions appropriées. La grenouille détecte des petites taches noires en mouvement qui ont à peu près la taille d'une mouche. Par conséquent, ce qui est transmis au cerveau de la grenouille par l'œil de la grenouille n'est pas un matériau brut (qui est supposé correspondre à la sensation pure) mais quelque chose de déjà élaboré. Ce que l'œil dit, écrit l'auteur de l'article, ressemble déjà beaucoup plus à un jugement de perception (sans le langage) qu'à une simple information sur du matériau sensoriel brut dont l'élaboration ultérieure est supposée. Autrement dit, la distinction sensationperception n'est pas toujours simple car la sensation est déjà une élaboration du réel.

La notion de seuil de sensation en est une preuve complémentaire. En effet, toute excitation n'est pas sensation, tout stimulus n'est pas sensible. Une excitation n'est sensible qu'au-delà d'un certain niveau nommé seuil absolu. La sensation se manifeste brusquement lorsque l'excitation atteint un seuil d'intensité. Toutefois, il faut distinguer seuil différentiel et seuil différentiel relatif. Si quelqu'un a en main un poids de cent grammes et si l'on ajoute quelques grammes, il en faudra au minimum cinq pour qu'il éprouve la sensation d'un accroissement de poids : le seuil différentiel est donc de cinq grammes. Si la personne porte un poids d'un kilo, il faut ajouter cinquante grammes pour qu'apparaisse la différence. Le seuil différentielest donc relatif au poids initial. Weber a tenté de montrer que cette loi du seuil différentiel relatif est constante. Fechner a, quant à lui, formulé l'hypothèse que la sensation croît comme le logarithme de l'excitation qui, elle, croît en progression géométrique. Mais après les critiques de Bergson, on peut dire au contraire, que la sensation ne traduit les variations du milieu qu'en les atténuant. L'organisme se protège en variant moins que le milieu. Car la sensation n'est pas le reflet passif du monde extérieur, elle joue un rôle biologique comme instrument d'adaptation de l'être vivant à l'univers.

La phénoménologie, tout particulièrement l'œuvre de Merleau-Ponty, qui se propose de décrire « ce qui apparaît ", c'est-à-dire le donné réellement vécu, affirme que le «sentir est immédiatement communication vitale avec le monde». La sensation pure est un mythe et aucune expérience ne nous livre de sensations pures. Comme le dit encore Merleau-Ponty « la sensation n'est pas sentie ». Il insiste particulièrement sur le rôle du sujet vivant, du corps propre comme centre de perspective. L'organisation globale du champ sensitif est effectuée par le corps et le sujet en situation. "Je suis dans ma sensation et, étant dans ma sensation, je suis dans le monde». Il s'agit donc de tenter d'élucider cet «être dans ». "Cet être dans », écrit Michel Malherbe dans Trois Essais sur le sensible, «c'est le corps propre, propre puisque s'y ébauche une singularité, mais corps appartenant au monde qu'il habite et participant de l'être de ce monde qui est son extension, le réseau de ses potentialités ». Le monde est le lieu du corps et la sensation inscrit l'homme dans son corps et dans le monde. Dans La Phénoménologie de la perception, Merleau-Ponty écrit : «le sentir investit la qualité d'une valeur vitale, la saisit d'abord 
dans sa signification pour nous, pour cette masse pesante qu'est notre corps et de là vient qu'il comporte toujours une référence au corps [...] Le sentir est cette communication vitale avec le monde qui nous le rend présent comme lien familier de notre vie ». Pour Merleau-Ponty, le corps est « au monde » depuis toujours. Il est une unité corporelle qui voit, qui entend, qui sent : « être corps, c'est être noué à un certain monde [...], et notre corps n'est pas d'abord dans l'espace (en ce sens qu'il serait une chose dans le monde objectif), il est à l'espace », (comme l'on dit d'un enfant qu'il est venu « au monde »).

Ces quelques réflexions visent à montrer que l'on ne saurait penser la sensibilité sans une analyse, même rapide, des mécanismes de la sensation. Concluons en affirmant que la sensation ne peut être considérée comme pure passivité, elle exprime le rapport du sujet au monde. Les différentes espèces vivantes « construisent " par la sensation, le rapport qu'elles entretiennent avec leur environnement.

\section{De la sensation à la sensibilité}

21 Mais la sensation n'est pas la sensibilité. Si la sensibilité a, à l'évidence, un support physiologique et biologique, elle est aussi en relation étroite avec la culture (cf. ce que nous avons dit à propos de l'arc-en-ciel). On pourrait dire qu'elle est l'interface entre le biologique et le culturel.

Pour approfondir cette première affirmation, ouvrons un instant le dossier des enfants sauvages et arrêtons-nous un moment sur les cas avérés de zoanthropie, représentés dans l'ouvrage de référence en la matière: Les enfants sauvages. Mythe et réalité(UGE 10-18 éd. 1964) de Lucien Malson. Kamala, une fillette de huit ans est trouvée en Inde en 1920, dans une grotte de loups, en compagnie d'une autre, plus jeune, Amala.

"L'une et l'autre ont d'épaisses callosités à la paume des mains, aux coudes, aux genoux, à la plante des pieds. Elles laissent pendre leur langue à travers des lèvres vermillon, épaisses et ourlées, imitent le halètement et ouvrent parfois démesurément les mâchoires. Elles ont deux modes de locomotion: sur les coudes et les rotules pour les petits déplacements lents, sur les mains et les pieds pour aller loin et pour courir- du reste avec rapidité. Les liquides sont lapés et la nourriture est prise, le visage penché, en position accroupie. Le goût exclusif pour les aliments carnés conduit les fillettes aux seules activités dont elles sont capables: donner la chasse aux poulets et déterrer les charognes et les entrailles ".

Les comportements de ces fillettes sont identiques à ceux des loups au milieu desquels elles ont été élevées et qui leur ont servi de modèles. De même en est-il de Victor de l'Aveyron recueilli et « éduqué » par Jean Itard.

"Cet infortuné se trouvait bien inférieur à quelques-uns de nos animaux domestiques, ses yeux sans fixité, sans expression, errant vaguement d'un objet à l'autre sans jamais s'arrêter à aucun, si peu instruits d'ailleurs et si peu exercés par le toucher qu'ils ne distinguaient pas un objet en relief d'un corps en peinture; l'organe de l'ouie insensible aux bruits les plus forts comme à la musique la plus touchante; celui de la voix réduit à un état complet de mutité et ne laissant échapper qu'un son guttural et uniforme».

Ces deux extraits montrent combien la socialisation de l'enfant est indispensable, sinon il ne parle pas, reste frustre, insensible au monde qui l'environne et demeure étranger à toute culture humaine. "L'homme est par nature, un animal politique », déclare Aristote dans La Politique, "et celui qui est sans cité, naturellement et non par suite des circonstances, est ou un être dégradé, ou au-dessus de l'Humanité ». Affirmer que l'homme est un animal politique signifie que la «culture humaine » le précède, que le langage est déjà là et que, par conséquent, l'enfant naît dans un «bain de langage » qui le 
façonne, le culturalise. Aucun enfant n'échappe à cette règle d'être plongé dès avant sa naissance, dans une culture, une histoire, des traditions, des coutumes qui le précèdent et sont pour lui une manière d'appréhender le monde et les autres. Le non développement de la sensibilité et du langage chez les enfants sauvages en est, a contrario, la preuve.

La langue est ce qui structure fondamentalement l'individu et l'intègre dans la communauté humaine, la parole est le proprement individuel qui s'exprime à travers la langue. Mais en même temps, comme le dit Bergson, la conscience est écrasée par le langage. « Non seulement le langage nous fait croire à l'invariabilité de nos sensations, mais il nous trompe sur le caractère de la sensation éprouvée. Ainsi par exemple, quand je mange d'un mets réputé exquis, le nom qu'il porte, gros de l'approbation qu'on lui donne, s'interpose entre ma sensation et ma conscience. Je pourrais croire que la saveur me plaît alors qu'un léger effort d'attention me prouverait le contraire» (Essai sur les données immédiates de la conscience). Toute langue est donc bien un système de valeurs qui privilégie tel élément plutôt que tel autre.

On pourrait en donner une preuve complémentaire, en s'intéressant à la question du goût et de l'alimentaire. Dans son livre, l'Homnivore, Claude Fischler propose le menu improbable d'un restaurant improbable. Petit déjeuner (10h30 - 13h30): Salade de fruits exotiques - Spaghetti alla carbonara - Soupe à l'oignon avec ses toasts - Thé glacé; Dîner (15h-19h30): Salade de saison - Escalopes de saumon sauvage à la moelle de bøuf - Consommé du Barry - Sorbets - Cappucino - Croissants au beurre - Liqueurs ou Kir royal. « Démonstration par l'absurde: tout, dans cette carte, est aberrant, au moins pour un individu de culture française » écrit l'auteur; chaque culture procède, en effet, d'un classement explicite élémentaire. Elle désigne les catégories du «comestible» et du «non comestible», du « pur » et de l'« impur» (porc chez les Musulmans). On pourrait, d'ailleurs, reprendre des expériences de la vie courante, où une personne trouve un plat excellent et devient malade lorsqu'elle apprend ce qu'elle a mangé (du cheval en Grande Bretagne - du chien ou du chat en Europe etc.) Autrement dit, nous ne consommons pas tout ce qui, dans notre environnement est biologiquement comestible. La complexité et la variabilité des mœurs alimentaires butent en réalité devant une accumulation de mystères. Claude Fischler ajoute : « Avant d'aller plus loin, il faut mettre en avant un élément décisif : homo sapiens est une espèce caractérisée par un néo-cortex hautement développé. Il n'est pas étonnant que les facteurs cognitifs ou idéals jouent un rôle extrêmement important dans la manière dont l'homme s'ajuste à son environnement, particulièrement en matière de choix alimentaire. La réponse à notre question initiale réside donc probablement en grande partie dans le mangeur et non pas seulement dans ses aliments; dans sa pensée, ses représentations, et non pas seulement dans son métabolisme [...] L'analogie entre langue et cuisine, banale depuis Lévi-Strauss, s'impose ici : tous les humains parlent une langue, mais il y a un grand nombre de langues différentes ». Depuis Lévi-Strauss, les analyses des exigences formelles du culinaire rappellent les contraintes des règles de syntaxe et de grammaire du langage, et certains repas apparaissent (cf. plus haut) comme des distorsions risibles ou des charabias insupportables.

On peut donc dire que la sensibilité humaine est orientée, conditionnée par le langage qui spécifie les choix d'une culture donnée. Sans le langage, il serait impossible à la sensibilité de trouver la moindre expression, mais, simultanément, le langage détermine la sensibilité, la limite et la délimite. Dans notre culture, on peut s'apercevoir assez facilement que le sens privilégié est la vue, au détriment des autres sens. On le voit, en particulier dans la difficulté à nommer les odeurs. S'il existe, par exemple, des mots pour 
désigner les couleurs, la désignation des odeurs est toujours métaphorique, allusive. Dans la dégustation d'un vin, les couleurs peuvent assez vite se repérer. Il en va tout autrement de l'odeur (on comparera avec l'odeur de sous-bois, avec les parfums de fruits rouges, odeur foxée, sauvage etc.) La culture et le langage donnent des « outils » à la sensibilité, mais en privilégient certains (la vue par exemple - le choix de certains mots etc.) La culture construit ainsi un ordre de l'univers qui est bien antérieur à l'individu, dans lequel il plonge par sa naissance et son éducation.

\section{L'éducation à la sensibilité ou « les mots pour le dire »} «qui perd, gagne?». Pour le montrer, reprenons un texte de Michel Serres écrit en préface à $L$ 'Oeuf transparent de Jacques Testard :

L'homme est l'être qui perd ses fonctions, ses instincts et qui abandonne l'immédiateté de son adaptation au monde. L'enfant met très longtemps à pouvoir s'insérer dans son milieu, à la différence de l'animal, d'où l'indispensable éducation. Mais ce qu'il perd en immédiateté, il le gagne en sensibilité : la main n'est plus une patte engluée dans sa fonction, elle devient instrument de connaissance. La bête, par son instinct, est en prise directe avec l'environnement. L'homme, au contraire, naît "nu », « livré » au monde ; «exposé» aux adultes, "à la merci» de l'autre. Le mythe platonicien du livre du Protagoras raconte comment l'insouciance d'Epiméthée laisse l'homme nu dans un monde hostile et menaçant. Platon montre comment le «souci philanthropique » de Prométhée procure aux humains l'intelligence, puisque le dieu dérobe le feu à Héphaïstos et à Athéna. Héphaïstos dieu forgeron, dieu boiteux, métamorphose au moyen du feu la matière tout entière. Athéna, déesse sortie toute armée du cerveau de Zeus, son père, représente le feu de l'intelligence conceptuelle. Par elle, s'ouvre la voie de l'« homo sapiens ", tandis qu'Héphaïstos ouvre celle de l'«homo faber». Ce mythe signifie la prise de possession du monde. Si l'homme perd le rapport primitif de son adaptation au monde, il gagne par le feu de son intelligence pratique et conceptuelle, la possibilité d'adapter le monde à lui-même et à ses besoins. Inversion donc du rapport initial; perdre son adaptation immédiate au monde signifie perdre tout ce qui délimitait chez l'homme ses potentialités et tout particulièrement sa sensibilité; la vue se transforme, l'ouie se métamorphose, le toucher devient caresse. La perte des poils, de la peau épaisse, de la corne des pieds, des mains libère la sensibilité. 
30 «Que tu es donc sensible!» entend-on parfois. N'est-ce pas là précisément l'expression même de la perte des protections adaptatives, de la carapace ou de la cuirasse tutélaire? La sensibilité, en ce sens, ne serait pas un "plus », mais plutôt un "manque ", une "perte», une «carence». Mais, en même temps, cette sensibilité est risque parce qu'exposition aux influences extérieures qui peuvent être analysées par le sujet comme dangereuses pour lui. Le sujet qui s'expose, qui s'ouvre à la sensibilité, perd ce qui dans l'instinct le protège. Le sujet est ici mis à vif, livré à l'autre. Par conséquent, perdre sa carapace signifie être " en creux », créer en soi un espace du possible, accepter la carence d'une non-plénitude. L'ouverture qui jusqu'à ce jour a caractérisé l'espèce humaine est le moyen d'entrer dans ce rapport inversé d'une adaptation du monde à l'homme. L'ouverture est aussi expérience: c'est-à-dire in-habileté (experitus = inhabile). La sensibilité est ouverture à l'expérience, au nouveau, à des domaines où le sujet est inhabile et par conséquent où il doit apprendre. Il en a sans doute été ainsi dans le domaine de l'alimentaire où l'expérience de l'omnivore a été d'accroître sa sensibilité à tous les aliments ; ce qui lui a permis de survivre dans certaines circonstances, mais aussi de se détruire dans des expériences tragiques (champignons par exemple). La sensibilité qui se développe de plus en plus avec la capacité humaine de distanciation avec le monde, permet d'affiner de plus en plus notre connaissance des choses et des autres.

31 Si l'homme perd, s'il est "poreux », cela favorise le déploiement de sa sensibilité. Toutefois cela n'est, au fond, que la condition de possibilité de ce développement. L'éducation joue un rôle essentiel dans cet apprentissage. Que faut-il entendre par là? Quels sont les lieux de cette éducation? Remarquons d'abord que le premier lieu est la famille, qui pour partie initie à la sensibilité et au langage des sens ; on désigne à l'enfant le propre et le sale («c'est caca»), le beau et le laid, le comestible et l'immangeable. Le langage lui indique les «bonnes " manières. Françoise Loux, dans Le corps dans la société traditionnelle, montre comment le corps est façonné depuis la naissance et par toute l'éducation familiale: " corps rigide de tous les jours, corps libre de la tendresse ; corps vêtu de l'apprentissage à la condition d'homme, corps nu des retrouvailles sexuelles avec la nature $»$.

Mais très vite d'autres instances, d'autres institutions vont prendre le relais. L'école bien sûr, avec sa discipline, avec l'apprentissage des «civilités » (depuis Erasme avec sa fameuse De la civilité puérile), de la pudeur, de la façon de se conduire en public. L'enfant "bien élevé » est celui qui sort de la grossièreté naturelle et de ses manifestations spontanées. Il est celui qui entre dans la culture et s'ouvre à toutes ses réalisations : musicale, picturale etc. L'école se veut lieu d'éducation artistique, ce qui n'est pas sans poser de problèmes, en particulier celui de savoir quelle place accorder à ces enseignements. D'autre part, les centres sociaux, les maisons pour tous, les écoles de dessin, les cours privés offrent aux enfants et adolescents des cours, hors temps scolaire, d'éducation artistique. Le champ de possibilités est sans doute limité et socialement orienté; peu d'éducation au goût (parfum-arôme etc.) par exemple. De ces grandes instances institutionnelles, on peut dire qu'elles ont une action directe, qu'elles visent une action immédiate et repérable $d^{\prime}$ ' apprentissage » de la sensibilité, comme en témoigne une décision du ministre de la Jeunesse et des Sports qui propose une expérience pédagogique dans quarante villes, en libérant trois après-midi par semaine de cinq jours, pour des activités artistiques ou sportives.

33 Toutefois, de manière beaucoup plus subtile, une éducation indirecte de la sensibilité se développe. Nous sommes façonnés, en effet, par les pratiques sociales. R. Arnheim dans La 
pensée visuelle (Flammarion 1976) écrit que « de nos jours, un profane reconnaît sans difficulté des hommes ou des animaux dans des tableaux impressionnistes qui, il y a quatre-vingts ans, apparaissaient comme des assortiments de taches de couleur dénués de toute signification ». La pratique sociale du "miroir», son utilisation quotidienne est radicalement autre que celle des hommes de la Renaissance qui venaient de découvrir à Venise le miroir parfait avec utilisation d'une couche de mercure entre deux plaques de verre. De nos jours, on notera que notre civilisation de la vitesse modifie nos perceptions sans que nous nous en rendions compte. L'art du cinéma, par exemple, en est transformé. Nous sommes forcés d'interpréter les images de plus en plus vite. Dans les films anciens, même parlants, l'action progresse avec lenteur, le jeu des acteurs nous paraît parfois plein d'une grandiloquence accablante. Chaque geste est appuyé, suivi, expliqué par la caméra. Une actrice ne changeait quasiment jamais de robe au cours d'un film, sinon le public ne l'aurait plus reconnue. Deux séquences étaient séparées par de lourds signes de ponctuation, des fondus-enchaînés ou des silences noirs. Le spectateur contemporain "décode " des successions frénétiques de plans, projetés sans transition, accepte les changements d'acteurs pour tenir le même rôle (séries américaines: Santa Barbara), comprend sans difficulté des séquences faites au total de cinq ou six images et qui durent un quart de seconde. Le monde dans lequel vit l'homme contemporain conditionne donc sa sensibilité. Il est en ce sens un véritable éducateur. Il oblige à traiter, à sélectionner, à interpréter dans leur contexte les phénomènes qui le traversent. La publicité en est un exemple flagrant. Les publicités des années cinquante nous paraissent désuètes et on peut se demander si un humain vivant au cours de ces mêmes années aurait « immédiatement » compris les publicités des années quatre-vingt-dix.

Mais surtout, l'éducation à la sensibilité, qu'elle soit directe (institutionnelle) ou indirecte (nous sommes «éduqués » par notre environnement), est avant tout le moyen de donner "les mots pour le dire ", pour reprendre le titre du livre de Marie Cardinal. L'expérience de la dégustation du vin le laisse entrevoir: si les mots manquent, il est difficile d'apprécier pleinement un vin. Au contraire, les mots permettent de sentir davantage, par leur capacité à différencier les couleurs, les parfums, les arômes, les goûts. Les stages de dégustation sont-ils autre chose qu'un apprentissage «des mots » pour dire le vin, pour dire la sensibilité au vin: au point de vue visuel (couleur - limpidité - fluidité effervescence), olfactif (odeurs élémentaires, arômes, puissance, qualité) gustatif (sensations dominantes, caractères, longueur persistante, "fin de bouche »). Mais aussi pour «filer les métaphores » : comparer le vin à une robe, étudier la «jambe » du vin, dire sa nature virile, entrer dans la poétique du vin. Sans oublier d'ailleurs le sens étymologique du grec «poien » qui veut dire fabriquer : en ce sens le poète est celui qui par les mots fabrique le monde, le construit, le rend vivant... Les mots sont donc un moyen de plonger au cœur du sensible lui-même en laissant résonner sa propre sensibilité.

L'éducation à la sensibilité crée donc un autre rapport avec l'objet qui n'est plus seulement saisi dans son instrumentalité, dans son utilité ou son efficacité, c'est-à-dire dans un rapport de domination, mais dans sa dimension esthétique, c'est-à-dire dans un rapport humanisé qui fait sens pour l'homme, qui «meuble» l'espace de vie, l'environnement humain. L'objet, par la sensibilité, vient habiter l'humain. Enfin, l'éducation à la sensibilité fait «boule de neige» - pourrait-on dire - car plus on est éduqué, plus on est sensible et plus on est sensible à tel domaine, plus on l'est à d'autres. Les mots permettent de dire la sensibilité dans la peinture, la musique, la littérature... 
L'éducation à la sensibilité est donc ouverture à la multiplicité des possibilités de la sensibilité.

Nous avons essayé de montrer comment la sensibilité est à l'interface de la sensation avec son support physiologique et biologique, de la culture et du langage qui valorisent, organisent le monde dans " une métaphysique cachée ». L'éducation à la sensibilité est donc essentielle puisqu'elle permet d'appréhender son propre rapport au monde et de l'habiter dans toutes les dimensions de son être.

\section{RÉSUMÉS}

La sensibilité est au croisement de la sensation avec son support physiologique et biologique d'une part, de la culture et du langage qui valorisent et organisent le monde en une «métaphysique cachée», d'autre part. L'éducation à la sensibilité doit se comprendre avant tout comme apprentissage d'une perte, comme abandon d'un déjà construit pour que l'éduqué puisse s'ouvrir à d'autres expériences et à la reconnaissance de nouvelles sensations.

We can define sensibility where sensation meets on one band with its physiological and biological support and on the other hand when it meets with the culture and language that organize and make the world attractive into a "hidden metaphysics". Educate to sensibility bas to be understood above ail as learning about loss, as an abandonment of something already built so that the educated person could open up to new experiences and to the recognition of new sensations.

\section{INDEX}

Mots-clés : langage, sensation, sensibilité

Keywords : language, sense, sensibility

\section{AUTEUR}

\section{JEAN-BERNARD PATURET}

Maître de conférences, Université de Montpellier III 